\title{
Internet based post-graduate course in spectacle lens design
}

\author{
Mo Jalie
}

Mo Jalie, "Internet based post-graduate course in spectacle lens design," Proc. SPIE 9289, 12th Education and Training in Optics and Photonics Conference, 92891N (17 July 2014); doi: 10.1117/12.2070528

SDIE Event: 12th Education and Training in Optics and Photonics Conference, SPIE. 2013, Porto, Portugal 


\title{
Internet based post-graduate course in spectacle lens design.
}

\author{
Professor M Jalie \\ University of Ulster
}

\begin{abstract}
The complexity of spectacle lenses has increased enormously over the last three decades. The advent of aspheric lenses for the normal power range and the, now commonplace, progressive lenses for the correction of presbyopia, are just two examples of $21^{\text {st }}$ Century technology. Freeform surfaces are now employed to personalize lenses to wearer's needs and these may be both progressive and atoroidal in nature. At the same time, optometry has taken a sideways step from optics and physics into a more general primary health care profession with an ever-increasing amount of biological and medical content added to an already brimming curriculum, hence the need for persons without optometry training to undertake the study of spectacle lenses.
\end{abstract}

Some years ago a post-graduate course was designed for opticians who had a good grasp of mathematics and the ability to pay close attention to detail in the lengthy trigonometric ray-tracing techniques employed in lens design calculations. The year-long course, is undertaken by distance learning, and has been undertaken via the internet by students from many countries around the world. Final assessment is by means of examination held by the Association of British Dispensing Opticians and takes the form of two three-hour papers, Paper One consisting of the determination of the aberrations of a spectacle lens by accurate trigonometric ray tracing and the second, a general paper on the optics of ophthalmic lenses. It leads to the professional qualification, ABDO (Hons) SLD.

Keywords: Spectacle lens, back vertex power, off-axis performance, oblique vertex sphere power, course material, delivery, support, submission, assessment, Association of British Dispensing Opticians.

It has always puzzled me as to why so few physicists have given any serious attention to the humble spectacle lens, surely, the commonest optical instrument in the modern world! I well remember, many years ago, a colleague from the physics department who had then, just recently, obtained his Masters with a thesis on aspheric optical elements, remarking to me, "a spectacle lens has only two surfaces - what on earth can you do with it?" The world of optometry, which is concerned with the correction of defects of the human eye, is very concerned with spectacle lenses, the complexity of which has increased enormously over the last two decades. The advent of aspheric lenses for the normal power range and the, now commonplace, progressive lens for the correction of presbyopia are just two examples of recent technology. Freeform surfaces are now employed to personalize lenses to wearer's needs and these may be both progressive and atoroidal in nature and can be designed in real time when the order from the eyecare practitioner is received by the ophthalmic lens manufacturing laboratory. At the same time as the need for greater technical understanding of the modern spectacle lens, optometry has taken a sideways step from optics and physics into a more general primary health care profession with an ever-increasing amount of biological and medical content being added to an already brimming curriculum. This has caused a major shift in the dispensing of spectacle lenses in the United Kingdom from optometrists to dispensing opticians.

So, what is different about the spectacle lens from almost all other optical elements? Firstly, a spectacle lens corrects an ametropic (out-of-focus) eye by arranging for the back vertex focus of the lens to coincide with the eye's far point (Figure 1). Thus spectacle lenses are numbered, not with their equivalent power as is usual with other optical elements, but instead, with their back vertex powers. Secondly, two thirds of ophthalmic prescriptions in the developed world include a correction for ocular astigmatism, so the prescription for the spectacle lens contains both a spherical and a cylindrical correction together with an axis direction for the correcting cylinder. The lens specification can be given in three separate forms, two 
alternate sphero-cylindrical forms or as a crossed cylinder form which expresses the minimum and maximum powers of the correction.

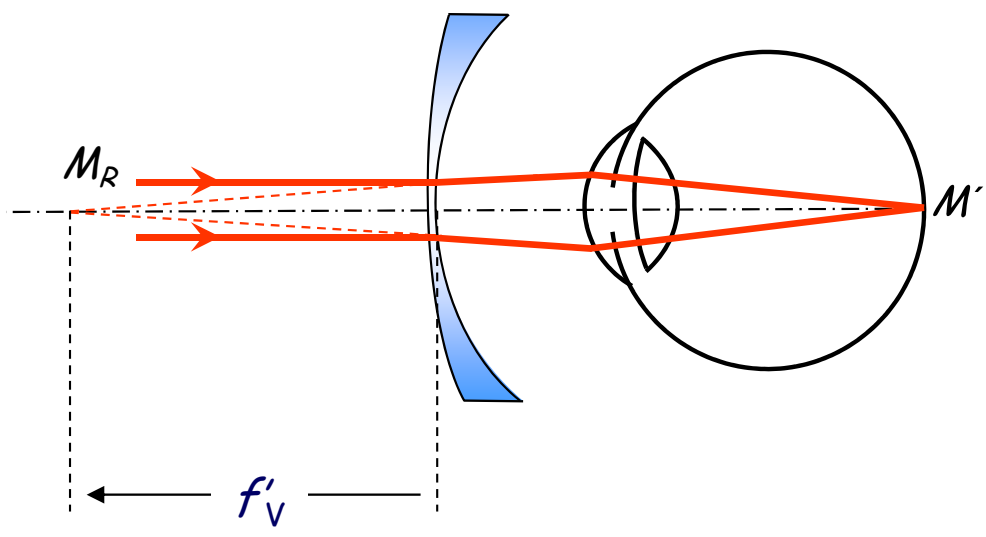

Figure 1 The correction of myopia by a spectacle lens.

The function of the spectacle lens is to bring incident parallel light to a focus at the far point, $M_{R}$, of the eye and, thus, to a focus on the macula, $M^{\prime} . \quad f_{\mathrm{V}}^{\prime}$ is the back vertex focal length of the lens.

For example, the specification $+2.00 \mathrm{DS} /+0.75 \mathrm{DC} \times 70$ might also be expressed as either $+2.75 \mathrm{DS} /-0.75$ DC x160 or as +2.00 DC x $160 /+2.75$ DC x 70 . In my experience, most physicists find this somewhat confusing to begin with!

Perhaps the biggest difference lies in the way in which the off-axis performance of a spectacle lens is judged. In spectacle lens design we are not dealing with a stationary eye. The eye rotates in its socket behind the spectacle lens in order to view off-axis objects. The eye's own pupil limits the size of the zone of the lens which can be used at any one time so that in the design of a spectacle lens we are interested in narrow obliquely incident pencils of light, the chief ray of which always passes through an assumed point about which the eye is supposed to rotate, the eye's centre of rotation (Figure 2). Thus, the spectacle lens designer is, normally, only interested in the four aberrations, oblique astigmatism, curvature of field, transverse chromatic aberration and distortion.

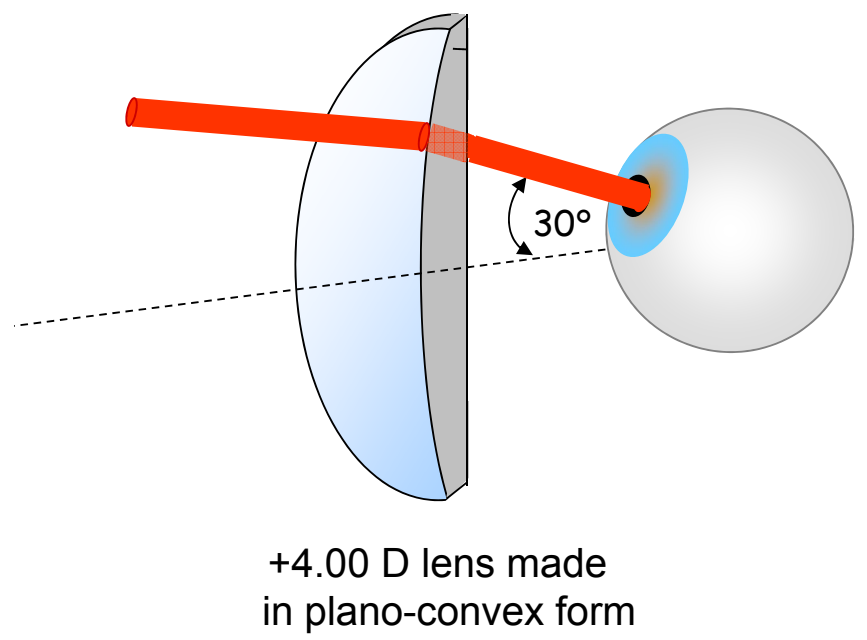

Figure 2 Use of the spectacle lens by a rotating eye.

It is assumed that the eye rotates about a single point close to the mechanical centre of the globe. This point is called the eye's centre of rotation. In the figure, the eye has rotated $30^{\circ}$ upwards about this point. 
Oblique astigmatism is considered to be the worst enemy of the spectacle wearer and, generally, must be eliminated, or at least, reduced to a very small quantity. If oblique astigmatism is completely eliminated, there is usually an error between the Petzval curvature of the image plane and the retina. This is often referred to as power error. Both astigmatism and power error are clearly indicated in the usual field diagram (Figure 3) which the spectacle lens designer uses to illustrate the off-axis performance of the lens. The field diagram illustrates the variation in tangential and sagittal oblique vertex sphere powers as the eye rotates away from the optical centre of the lens. Three typical field diagrams are given in Figure 3 which illustrate the off-axis performance of (i) an ideal spectacle lens of power +4.00 D, (ii) a $+4.00 \mathrm{D}$ lens made in planoconvex form with the plane surface facing the eye and (iii) a point focal $+4.00 \mathrm{D}$ lens made with a front surface of power $+9.50 \mathrm{D}$, which is the form required to eliminate oblique astigmatism over a field angle of $40^{\circ}$ on either side of the optical axis. Figure 3(i) shows the ideal performance for the lens, the tangential (T) and sagittal (S) oblique vertex sphere powers should remain the same as the back vertex power of the lens, $+4.00 \mathrm{D}$. It is impossible to make such a lens when we are restricted to a single optical element! Considering the plano-convex form illustrated in Figure 3(ii), it can be seen that if such a form was employed, then the tangential and sagittal oblique vertex sphere powers increase as the eye rotates away from the centre of the lens. At $30^{\circ}$ rotation the sagittal power has increased to about $+4.20 \mathrm{D}$ whereas the tangential power has increased to about $+5.20 \mathrm{D}$. The difference between these two values is the oblique astigmatic error, +1.00 D, exhibited by the lens for the $30^{\circ}$ zone. Figure 3(iii) illustrates the off-axis performance for a spectacle lens which has been bent into a form which exhibits zero astigmatism across the field. Such a form is described as point focal and although there is zero astigmatism, the lens still exhibits a small power error of about $0.20 \mathrm{D}$. The errors exhibited by poorly designed spectacle lenses are clearly, too large to be ignored!

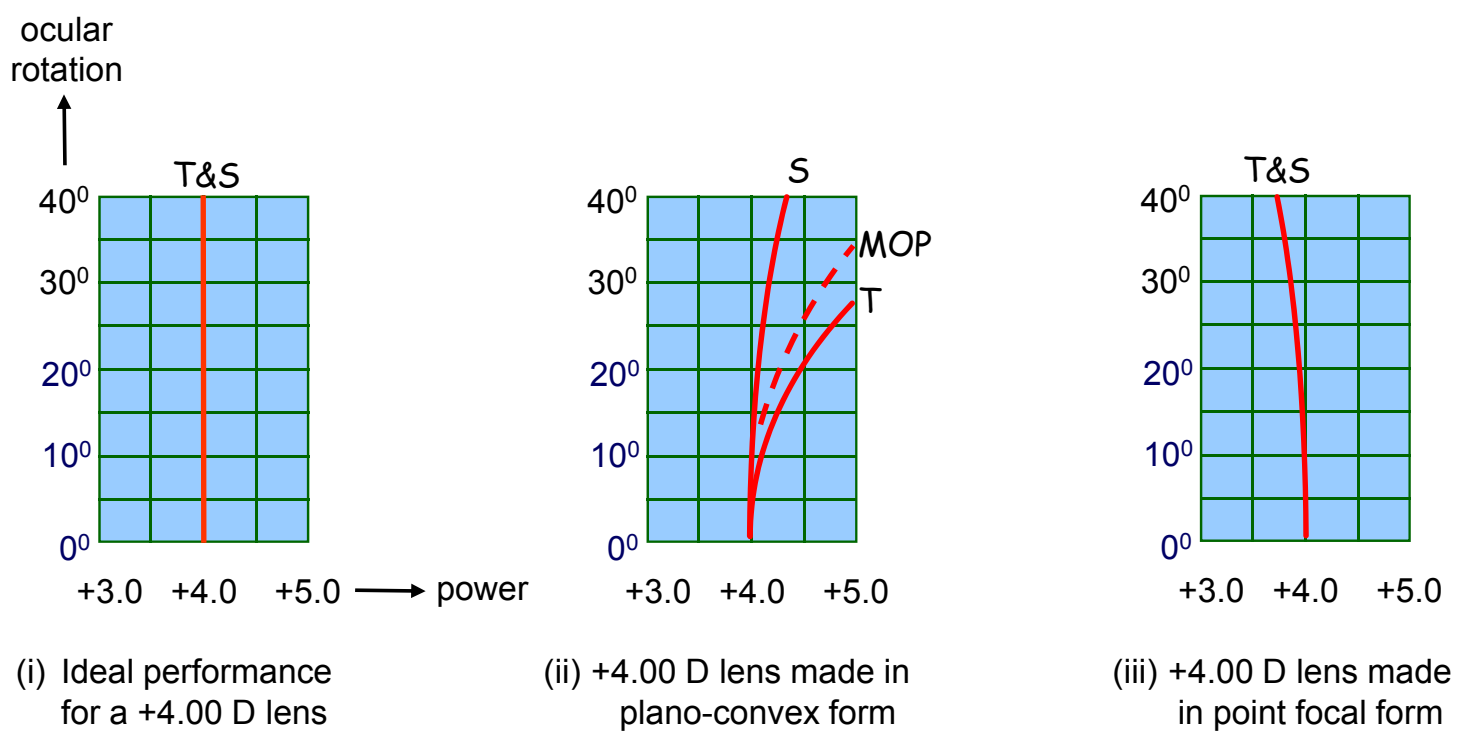

Figure 3 Field diagrams for spectacle lenses.

i) Field diagram for an ideal $+4.00 \mathrm{D}$ lens. The lens power should remain $+4.00 \mathrm{D}$ for all zones of the lens.

ii) Field diagram for a $+4.00 \mathrm{D}$ lens made in plano-convex form illustrating the poor off-axis performance of this form. The oblique astigmatic error at $30^{\circ}$ is seen to be $+1.00 \mathrm{D}$ and the mean oblique power is +4.75 $\mathrm{D}$, there being a power error of about $+0.75 \mathrm{D}$.

iii) Field diagram for a point focal $+4.00 \mathrm{D}$ lens. The front surface power of the lens is $+9.50 \mathrm{D}$ for which form, the oblique astigmatic error is zero. The mean oblique power at $30^{\circ}$ is about $+3.80 \mathrm{D}$ and the power error is $-0.20 \mathrm{D}$.

For at least forty years, the professional body for dispensing opticians in the United Kingdom, The Association of British Dispensing Opticians, has run an honours course in the harder aspects of spectacle lens construction, design and performance leading to the qualification, FBDO(Hons) SLD. This postgraduate course was designed for dispensing opticians who had a good grasp of mathematics and the ability to pay close attention to detail in the lengthy trigonometric ray-tracing techniques employed in lens design calculations. The year-long course, originally re-written by myself in the 1970 s, was undertaken by distance 
learning, a mode of teaching in which the Association has a great deal of experience, with students in many countries around the world. Final assessment is by means of examination with two three-hour papers being undertaken, Paper One consisting of the determination of the aberrations of a spectacle lens by accurate trigonometric ray tracing and the second, a general paper on the optics of ophthalmic lenses. (The syllabus outlined below gives full details of the course material.)

At the turn of the last century, the Association asked me, under the auspices of OptNet.org.uk, to fully revise the course and take over its delivery, using either a traditional distance learning mode (exchange of coursework by written correspondence) or web-based means for those participants who had access to the internet. OptNet had pioneered a three-year distance learning course for opticians which was delivered entirely by CD using PowerPoint and Flash animations. OptNet, whose personnel were all UK academics with lengthy teaching experience on optical courses had developed the technology to enable its optician's course to be delivered entirely by the internet. It was foreseen that internet delivery of the honours course in Spectacle Lens Design would make it easier and more convenient for overseas students to undertake this post-graduate training.

It was agreed that the Association would continue to conduct the examinations leading to an honours qualification, and also that persons who were not members of the Association would be allowed to undertake the examination and if successful, to obtain the qualification, $A B D O$ (Hons) SLD. It was hoped that the course would also prove to be attractive to optical technicians and engineers who wished to consider the theory of spectacle lenses in greater detail.

In 2002 the new course was offered by OptNet having been fully updated to include the theory and design of aspheric lenses. This lecture describes the course content and the method by which this post-graduate course is delivered via the internet.

\section{COURSE SYLLABUS SPECTACLE LENS DESIGN}

\section{Paraxial optics of ophthalmic lenses}

Paraxial ray tracing through lenses and lens systems - the general case Accurate transposition - special cases

Strong positive lenses of specified diameter and edge thickness

The spectacle lens in near vision - paraxial considerations

Trial lens design

Optical centration of distance and near portions of bifocal lenses

Binocular vision - ocular rotations

Iso-prism zone theory

Calculation of iso-V-differential prism zones

Reflections from spectacle lenses

Tinted lenses - terms relating to transmission, reflection and coatings

Spectral weighting functions

Luminous transmittance

Relative visual attenuation coefficients

Iseikonic lens design

Isogonal lens design

\section{Trigonometric ray tracing - general}

Introduction to ray tracing techniques

Ray tracing through plane and spherical surfaces

Ray tracing to determine spherical aberration

Ray tracing to detemine minimum spherical aberration forms

Aplanatic points

Field of view

Calculation of transverse chromatic aberration

Calculation of distortion 


\section{Spectacle lens design}

Third order theory DV and NV

Best form lenses - types and advantages of design philosophies

Ray tracing through spherical surfaces

Determination of the near path through spectacle lenses

Geometry of toroidal surfaces

Ray tracing through toroidal surfaces

Geometry of conicoidal surfaces

Higher order aspherical surfaces

Determination of the conicoid required to eliminate tangential error

Ray tracing through conicoidal surfaces

Design of spherical lenses

Design of toric lenses

Design of aspheric lenses

\section{Course Material}

The course comprises 32 units which are completed over a 40 week period. There is no pressure on the student to complete the course within this time frame, if necessary the student may take longer to complete and return the Assignments. Most students do complete the course within one 40-week period and take the examination, which is held at the end of the course in either June or December.

The main textbook employed for the course is my own Principles of Ophthalmic Lenses which contains the necessary theory including the mathematical routines required for tracing rays of light through spherical, toroidal and aspherical surfaces.

\section{Delivery of course material}

In traditional distance learning courses, assignments, CDs (or even the older floppy disk) are distributed by surface or air mail. For the Spectacle Lens Design course, when undertaken via the internet, all material required for study is sent as e-mail attachments. The student also receives a study programme mapping out their academic year and enabling them to keep a record of their achievement during the course.

\section{Online support}

One of the most significant advantages of communicating by the internet is the ability to hold online tutorials at virtually no cost. Questions from students may arrive simply as a query by e-mail, but in the same way as a query sent in by post, there may be an inconvenient delay from the time when the question arose, to the time when the answer is returned. Students generally feel that they receive a better service and are in closer rapport with their tutors when they are in direct touch with them in real time. This is possible today with the availability of free communication software such as SKYPE which allows

- $\quad$ simple text conversation

- audio conversation

- video conversation.

Needless to say, a sound card, microphone and speakers are required at both the tuor's and the student's workstation, for audio conversations, with the addition of a webcam for video conversations. Most workstations, (desktops and laptops), of course, already have these facilities built in. Until April of this year, Microsoft also offered MSN MESSENGER, its 14-year-old instant chat service but has now terminated this service. MSN Messenger users can now access Skype which it integrated in 2011. MSN Messenger allowed a live whiteboard to appear on the screen, that could be viewed by both the tutor and the student simultaneously, and upon which the tutor can write, draw and answer questions as though in a classroom situation with the student! Naturally, broadband connection is essential to obtain the best results from audio and video conversations. Appointments for connection via the internet are easily arranged by email or telephone. 


\section{Submission of work by students}

If the student is fluent in Microsoft WORD and its drawing packages they can submit their work for assessment entirely electronically. The tutor can easily open WORD documents and add marks and make comments directly on the submitted documents. Many students prefer to write their assignments by hand, adding any diagrams and graphs as necessary and then scanning the document into a file for submission by e-mail. Work submitted in this way can be opened to enable the tutor to mark and correct the handwritten pages which can then be returned to the student by e-mail.

\section{Return of marked assignments}

The tutor's task of returning work to the student via the internet is quite simple since the original submission from the student is already available in electronic form.

Model answers to the assignments are also sent with the marked paper, together with a detailed explanation of where any errors have arisen in the student's workings.

\section{Final Assessment}

Upon completion of the course, students undertake the Honours Examination in Spectacle Lens Design organised by the Association of British Dispensing Opticians. The examination consists of two, three hour papers, the first of which is, typically a single question asking the candidate to determine the oblique vertex sphere powers, transverse chromatic aberration and distortion for a given spectacle lens.

The second paper requires the candidate to answer six questions drawn from the syllabus outlined above Success in the examination leads to the award of an Honours Diploma in Spectacle Lens Design of the Association of British Dispensing Opticians, FBDO(Hons) SLD if the candidate is already a Fellow of the Association. For those candidates who are not Fellows, the qualification ABDO(Hons) SLD is awarded. 